\title{
Equalized Energy Consumption in Wireless Body Area Networks for a Prolonged Network Lifetime
}

\author{
Maryam El Azhari, Nadya El Moussaid, Ahmed Toumanari, and Rachid Latif
}

Ibn Zohr University, Agadir, Morocco

Correspondence should be addressed to Maryam El Azhari; maryam.elazhari@edu.uiz.ac.ma

Received 13 August 2017; Revised 26 October 2017; Accepted 6 November 2017; Published 20 December 2017

Academic Editor: Houbing Song

Copyright ( 2017 Maryam El Azhari et al. This is an open access article distributed under the Creative Commons Attribution License, which permits unrestricted use, distribution, and reproduction in any medium, provided the original work is properly cited.

\begin{abstract}
The phenomenal advances in electronics contributed to a widespread use of distributed sensors in wireless communications. A set of biosensors can be deployed or implanted in the human body to form a Wireless Body Area Network (WBAN), where various WBAN PHY layers are utilized. The WBAN allows the measurement of physiological data, which is forwarded by the gateway to the base station for analysis purposes. The main issue in conceiving a WBAN communication mechanism is to manage the residual energy of sensors. The mobile agent system has been widely applied for surveillance applications in Wireless Sensor Networks (WSNs). It consists in dispatching one or more mobile agents simultaneously to collect data, while following a predetermined optimum itinerary. The continuous use of the optimal itinerary leads to a rapid depletion of sensor nodes batteries, which minimizes the network lifetime. This paper presents a new algorithm to equalize the energy consumption among sensor motes. The algorithm exploits all the available paths towards the destination and classifies them with respect to the end-to-end delay and the overall energy consumption. The proposed algorithm performs better compared to the optimal routing path. It increases the network lifetime to the maximum by postponing routing of data via the most-recently used path, and it also maintains data delivery within the delay interval threshold.
\end{abstract}

\section{Introduction}

WBANs are considered one of the most recent research topics [1-3]. In fact, the patient can perform activities of daily living while physiological data are continuously monitored by medical centers. A WBAN is composed of several biosensors implanted or deployed on the body to measure medical parameters, such as body temperature, arterial pressure, and EEG Signal [3-8]. Once the aforementioned parameters are collected, they are transmitted to the base station where further analyses are taking place by the specialists. For this reason, the overall architecture of a WBAN is subdivided into three levels: the first level includes local data transmission from biosensors to the Personal Digital Assistant (PDA), which is located within the transmission range of the biosensor (in case the biosensors are linked by a star topology), or it can be retransmitted towards a relay biosensor using a multihops topology with a step of 2 . The relay biosensors are characterized by important energy resources and wide communication range. Moreover, they have the ability to maintain the power of transmission very low leading to minimal energy consumption, while the heating effect is kept inferior to a specific threshold defined by SAR [9] for a prolonged period of time. The PDA is usually powered with AA batteries and does not have the problem of limited energy resources. It forwards data towards the final destination through an intermediate level (level 2), which can be either a WIFI, 3G, 4G, WSNs, or a cellular network. The choice of the intermediate network is application-oriented, but it often tends to minimize the overall installation cost. Finally, the third level consists of a set of endpoints, such as the Personal Digital Assistant, linked to a mega database where patients records are stored and analyzed by specialists in the domain. In a spacious area like the hospital, several WBSNs with diverse mobility patterns coexist. They either follow a static pattern such as lying-down and sitting or a dynamic pattern, for instance, walking or running [10]. These WBSNs collaborate with a set of statically deployed 


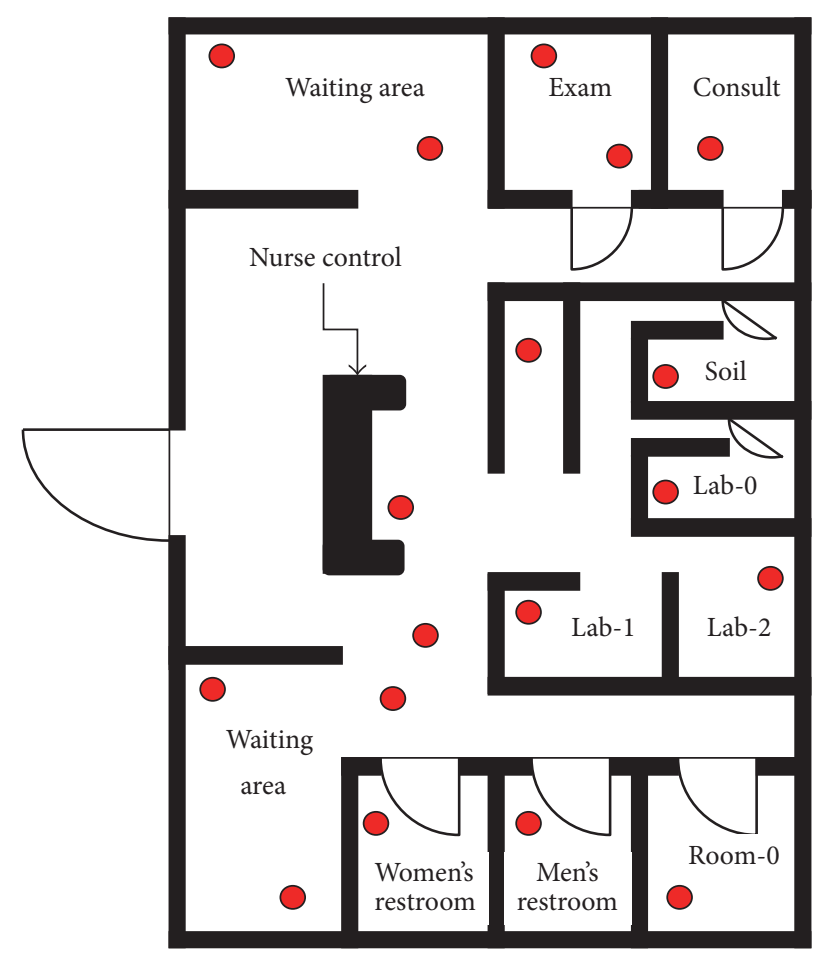

FIGURE 1: Example of relay sensors distribution in a hospital.

relay sensors (Wireless Sensor Networks) to deliver medical data. As can be seen from Figure 1, relay sensors (red dots) are scattered in a way to cover the different spots visited by the patient. The relay sensors are often called source nodes, to which a number of sensor nodes are added to insure sensors connectivity and multihop routing. In this work, we aim to enhance the performance of the WBANs in terms of prolonging the network lifetime. The emphasis is given to the second level composed of relay sensor nodes. Our new optimal routing mechanism consists in utilizing the mobile agent technology coupled with an equalized energy consumption schema. The latter takes into consideration the residual energy of each relay sensor in accordance with data collection method, which is proven to be efficient in terms of minimizing the overall energy consumption and increasing the network lifetime.

\section{Related Works}

In [11], a modified Stable Election Protocol (SEP), named Prolong-SEP (P-SEP), is presented to prolong the stable period of Fog-supported sensor networks. It consists in maintaining balanced energy consumption as all sensor nodes have the same probability to be selected as a cluster head. The experimental results showed that the proposed algorithm achieves much higher energy saving than the traditional one. In [12], the authors proposed an Energy-Efficient $k$-Coverage Algorithm (EEKCA), which constructs a network coverage model by utilizing the relative positions of nodes. EEKCA calculates the coverage expectation with a sector and gives the process to fulfill the coverage with the minimum number of sensor nodes. The simulation results showed that the proposed algorithm improves not only the coverage quality of the network but it also prevents the rapid depletion of sensor nodes energy, achieving though an extended network lifetime. Nevertheless, Wireless Sensor Networks algorithms are designed to tackle bigger size networks. Besides, the cluster-based architecture has been proven to be an inconvenient choice for WBAN [13]. Moreover, the previously mentioned algorithms did not take into account the sensor motes where the energy consumption does not respect the theoretical model of sensor node's battery depletion [14]. In [15], the authors reviewed WBAN communication architecture, security, and privacy requirements in WBAN. A novel biometric algorithm was designed in [16] based on biometric feature Electrocardiogram and Data Authentication Function in order to replace the traditional key generation techniques and attain security and privacy in WBANs. The simulation results showed that the proposed scheme outperforms symmetric encryption based technique DES and asymmetric encryption based algorithm RSA. Moreover, fewer resources were required during data transmission, which offered costefficient solution compared with the conventional approaches for WBAN security. In [17], a key generation algorithm using ECG feature biometric for WBAN security was proposed. The fast Fourier transform method was used to individually process the selected ECG datasets of diabetic patients. The simulation results showed that the algorithm provides a secured internode communication with a $25 \%$ energy efficiency in node transmission energy consumption for a WBAN system.

\section{Energy Consumption Model}

WSNs design is influenced by the sensor motes limited energies resources. In fact, sensor motes are powered with AA batteries, which can be either rechargeable or replaced when they run out of energy. However, this option is not always available, and sensors are discarded once their batteries are depleted. Sensors endowed with rechargeable batteries benefit from external energy resources such as solar power, and when they are replaceable, they are often nonaccessible as they are deployed randomly in furthest region. To this end, it is crucial to adopt a stingy energy consumption approach in order to increase the network lifetime to the maximum while guaranteeing application tasks completion. Many researches are carried out to deal with low-energy radios. In fact, various assumptions about radio modeling have been proposed and they are still in use to develop new optimal energy consumption paradigm for WSNs. In [18], the researchers defined three basic operations that consume energy batteries to a certain extent. Therefore, we distinguish between energy of acquisition which corresponds to the energy spent to convert the collected parameter to digital signal, energy of processing required to process the signal before proceeding to transmission, and energy of communication which corresponds to data transmission to a particular destination. The energy of communication represents the largest portion of the overall energy consumed by a sensor node in comparison with both energy of acquisition and energy of processing. The energy consumed by the transceiver can be theoretically modeled 


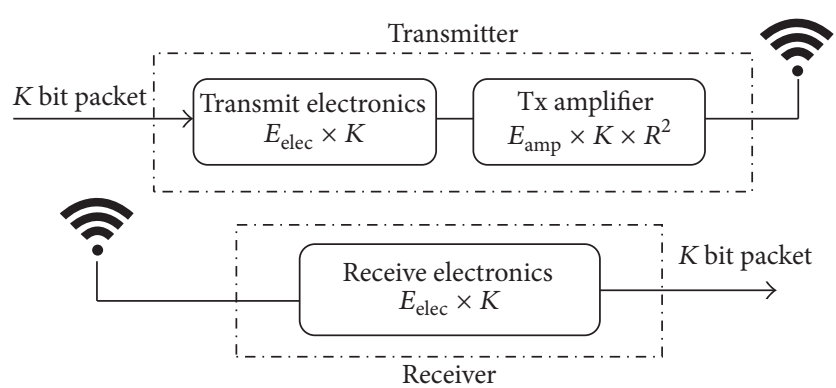

Figure 2: The theoretical energy consumption model of the transceiver.

as shown in Figure 2. The energy required to send a $k$ size data packet (in bits) is calculated in respect to the distance between the transmitter and the receiver and also the radio characteristics.

$$
E_{\mathrm{tx}}(k, d)=k \cdot\left(E_{\mathrm{elec}}+E_{\mathrm{amp}} \cdot d^{2}\right),
$$

where $E_{\text {elec }}$ is the amount of energy needed to run the transmitter or the receiver circuitry and $E_{\text {amp }}$ refers to the antenna gain at the transmitter and the receiver. Similarly, in order to receive $k$ data bits, the following amount of energy is consumed:

$$
E_{\mathrm{rx}}(k)=E_{\mathrm{elec}} \cdot k
$$

The transceiver model depicted above was customized to include extra sources of energy consumption such as the energy corresponding to low power sleep mode or idle listening for a message. However, this model remains an abstraction of the radio theoretical model, as the energy consumption of the most recent model depends on the functioning time during which the model is in active model (see Table 1). To this end, it is crucial to take into account the actual distribution of the energy consumption in order to give the most suitable solutions to a particular radio model.

\section{Mobile Agent Paradigm}

The mobile agent paradigm has been widely utilized for an efficient data management. In fact, data can be sensed and treated locally based on the portion of the code carried by the mobile agent when it is roaming the network. The mobile agent code size mainly depends on the measured parameters. In other words, as the number of these parameters increases up to a certain limit, the size of collected data evenly increases, which affects the overall energy needed to transmit or receive data. The mobile agent paradigm can be a packet including a preestablished path to collect data. The packet is retransmitted to the intermediate nodes in the vicinity until it reaches the destination node. The mobile agent can also be referred to as a mobile node or an actuator, capable of acting upon the physical environment. Such entities can be implemented to perform networking-related functionalities such as receiving, transmitting, processing, and relaying data towards the destination. They also roam the network to particular positions and proceed with data measurement in respect to the application requirements.
TABLE 1: Radio model characteristics.

\begin{tabular}{lcccc}
\hline Radio & TR1000 & TR1000 & CC1000 & CC2420 \\
\hline $\begin{array}{l}\text { Data rate (kbps) } \\
\text { Modulation type }\end{array}$ & OOK & ASK & FSK & O-QPSK \\
$\begin{array}{l}\text { Receive power } \\
(\mathrm{mW})\end{array}$ & 9 & 12 & 29 & 38 \\
$\begin{array}{l}\text { Transmit power } \\
\text { at } 0 \mathrm{dBm}(\mathrm{mW})\end{array}$ & 36 & 36 & 42 & 35 \\
\hline
\end{tabular}

In [19], the average energy consumption within WSNs has been highlighted when the mobile agent packet and the mobile agent sensor mote are implemented. As mentioned in [19], the mobile agent node approach shows a better performance in terms of energy consumption, as it reaches a maximum value equal to $0,080115 \mathrm{~mJ}$ (Sn2) versus $0,555522 \mathrm{~mJ}$ (Sn4) in case of mobile agent packet. This behavior was expected as the energy consumption model of sensor nodes depends on both data and code sizes but also the distance between the sender and the receiver. Thereby, the mobile agent packet size increases exponentially which explains the results obtained. The mobile agent packet needs to check the connectivity between two consecutive sensor nodes in the preestablished path, for there is no forwarding mechanism taking place. However, when considering the mobile agent node, data packets are aggregated locally. In other words, the sensor node communicates the same amount of data size every time it is interrogated by the mobile agent located within its communication range. Besides, the maximum energy consumption is formulated in respect to the communication range length of the sensor node. The mobile agent packet approach presents an important advantage concerning data transmission latency, which is reduced to $0,13 \mathrm{~s}$ compared with $8 \mathrm{~s}$ for the mobile agent node. Again, this behavior was expected as the mobile agent node roams the network with a particular speed $(\mathrm{m} / \mathrm{s})$ value to reach the following destination, which explains the delay being found. Based on the aforementioned results, we base our mobile agent approach choice on minimizing EPD factor (Energy Delay). The purpose is to give a trade-off balance between the energy consumption and the end-to-end delay. However, this choice will certainly serve some applications while giving undesirable performances to others. For instance, some application put much emphasis on the transmission delay. Hence, it is important to use a mobile agent where the latency does not overcome a specific threshold. Nevertheless, other applications focus on maintaining the network lifetime as long as possible, which makes the energy consumption the main problem to resolve. Thereby, the choice of the mobile agent approach remains application-oriented, determined by the user.

\section{MABMR: Mobile Agent Based Multipath Routing}

Many researches have been focusing on finding the optimal number of mobile agents to disperse in the network, but also the optimum itinerary to follow in order to maintain a minimal energy consumed and guarantee data delivery within 


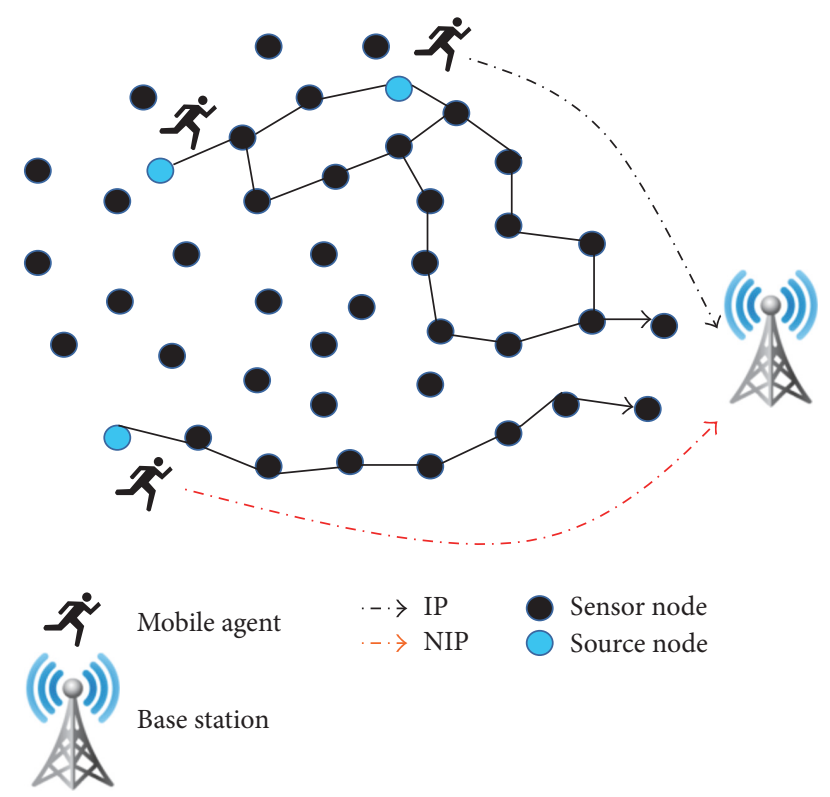

FIGURE 3: The mobile agent multiple itineraries towards the destination.

the delay interval threshold. However, the continuous use of the same itinerary for data collection leads to unbalanced energy consumption; therefore, the sensor nodes will run out of energy in a short period of time. The network lifetime often indicates the first sensor node experiencing a complete battery depletion. However our algorithm is valid for any network lifetime definition since it puts much emphasis on equalizing the energy consumption among sensors, thus extending the network lifetime for many rounds.

Our algorithm is based on exploring all the possible itineraries from source nodes to the base station in respect of data transmission delay, which does not overcome a predefined delay threshold (see Figure 3). These itineraries are classified in a way that paths including intersected nodes have the least ranking as they may create energy depletion holes in the network after a short time period of its use. Algorithm 1 is implemented to establish the mobile agent itineraries. It has a quadratic complexity $\left(\mathcal{O}\left(n^{2}\right)\right)$ and it starts off with defining all possible paths from the source nodes to destination node, that is, the base station. These paths are then filtered in a way that the path's delay that exceeds a delay threshold (measured in respect to the application type in use) is excluded from the overall paths set, and then the resulting set will be subdivided into two main subsets which are as follows: NIP (Nonintersected Paths) and IP (Intersected Paths). NIP $s_{i}$ of a source node $s_{i}$ designates all paths that do not intersect with any other path.

One has $P_{j}$ of a source node $s_{j}$ where $i \neq j$ (see Algorithm 2). The main purpose of this subdivision is to give priority to paths that exclude intersection nodes as they rapidly deplete energy. If the NIP set is not null, then the paths belonging to this set will be used successively as mobile agent itineraries, else the IP set will be selected and paths will be classified either horizontally or vertically in respect to the type of data to be collected. The vertical classification is taking
$\mathrm{CHS}_{s}$ : indicates the set of all possible paths of a source node $s$.

BS: indicates the base station.

$\mathrm{SN}$ : corresponds to the number of source nodes in the network.

Num_CHS : the number of paths in $\mathrm{CH}_{s}$ set.

$\mathrm{NIP}_{s}$ : the set of all non intersecting paths of the source node $s$.

$\mathrm{IP}_{s}$ : the set of all intersecting paths of the source node $s$. $\operatorname{Tr}\left(P_{i}\right)$ : a function indicating packet transmission delay corresponding to a path $P_{i}$.

for $s=1$ to SN do $\quad \triangleright$ Definition of $\mathrm{CHS}_{s}$

Define all possible paths towards the Base Station end for

for $P_{i}=1$ to Num_CHS $s$ do

if $\operatorname{Tr}\left(P_{i}\right) \geq$ DELAY_THRESHOLD then $\quad \triangleright$ $\mathrm{CHS}_{s}$ filtering

Suppression of $P_{i}$ path.

end if

end for

for $P_{i}=1$ to Num_CHS_s do $\quad \triangleright$ Definition of NIP $s$ and $\mathrm{IP}_{s}$

if $P_{i} \cap P_{j}=\emptyset$ then

$\forall P_{j} \in \bigcup \mathrm{CHS}_{k}$ where $k \in \mathrm{SN}, k \neq s$

Insert $P_{i}$ into $\mathrm{NIP}_{s}$ set.

else

Insert $P_{i}$ into $\mathrm{IP}_{s}$ set.

end if

end for

Algorithm 1

SUB_NIPs: indicates a sub set of $\mathrm{IP}_{s}$ non intersected paths.

SUB_IPs: indicates a sub set of $\mathrm{IP}_{s}$ intersected paths. PERIODIC_COLLECT: Periodic data collection

EVENT_COLLECT: Random Data collection based on event occurrence or user request. use paths of SUB_NIPs successively.

if NIP $\neq \neq \emptyset$ then

Use paths of $\mathrm{NIP}_{s}$ set successively.

goto : end $\quad \triangleright$ The end of the algorithm else

if NIPs $=\emptyset \& \&$ IP $s \neq \emptyset$ then

if EVENT_COLLECT then

goto : end

use IPs set paths successively.

end if

if PERIODIC_COLLECT then

if $\operatorname{SUB} \_N I P s \neq \emptyset$ then

goto : end

use paths of SUB_NIPs successively.

else

use paths of SUB_IPs successively. goto

: end

$$
\begin{aligned}
& \text { end if } \\
& \text { end if }
\end{aligned}
$$

end if

fin: The end of the algorithm

end if 
place when data is collected upon reception of a request or after the occurrence of an event. In this case of study, we believe that the maximum number of events occurrences during a time interval $T$ does not exceed 1 . Thus, the order of the one-dimensional source nodes paths vector $V_{T}$ will vary from 0 to $\mathrm{SN}$, where $\mathrm{SN}$ indicates the number of source nodes. The horizontal classification corresponds to a periodic data collection, meaning that, for each interval $T, V_{T}$ is already established and its order is constant and equal to $(1, \mathrm{SN})$. The structure of an intersected path is defined in accordance with other source nodes paths, the intersecting points, and the degree of their occurrences:

$$
\left\langle\left\langle P_{k, j}\right\rangle, \bigcup\left\langle i, \alpha_{i}\right\rangle\right\rangle
$$

where

(i) $k \in \mathrm{SN}$ and $i$ is an intersection point, $i \in \mathrm{ID} \_\mathrm{SN}, i \neq 0$;

(ii) SN is the set of source nodes and ID_SN is the set of sensor node identifiers;

(iii) num_IPs corresponds to the size of $\mathrm{IP}_{s}$ set;

(iv) num_CHS_s is the size of all paths;

(v) Current_ID_SN indicates the current source node identifier;

(vi) $\alpha \in\left\{0, \ldots, \sum\right.$ num_CHS_s $\}, s \neq$ Current_ID_SN;

(vii) $j \in$ num_IPs and $k \in \mathrm{SN}$.

5.1. Realistic Model. When data are collected randomly, the IP set of a source node is classified vertically, that is, in respect to other paths of the same source node. However, when period data collection is taking place, IP set is classified in respect to IP sets of other source nodes. To this end, we defined the coefficient of each path based on the energy consumption model. The formula that corresponds to the theoretical model is defined as follows:

$$
\begin{gathered}
\left\{\sum_{j=1}^{\mathrm{ID} \_\mathrm{SN}} a^{\max \left(\alpha_{j}\right)-1}+\sum_{j \in\left\{1 \cdots \mathrm{ID} \_\mathrm{SN}-1\right\}} a^{\alpha_{j}}\right\}, \quad \max \left(\alpha_{j}\right) \neq 0 \\
\sum_{j \in\left\{1 \cdots \mathrm{ID} \_\mathrm{SN}-1\right\}} a^{\alpha_{j}}, \quad \max \left(\alpha_{j}\right)=0, a \in \mathbb{N}-\{0,1\} .
\end{gathered}
$$

As depicted in the above formula, paths including intersecting nodes with the highest degree have lowest ranking compared with those with no intersecting nodes or where the degree of the intersecting node is null.

5.2. Theoretical Model. As previously mentioned, the theoretical model depends mainly on the distance between the sender and the receiver but also the exchanged data length. The latter can be interpreted as the order of sensor node in the path. In fact, the retransmitted data gets bigger in terms of bit size; therefore, the closer a sensor node is to the destination, the higher the order in the path is. For this, we define the
TABLE 2: The weighting factors parameters value.

\begin{tabular}{lc}
\hline$C_{r}(\mathrm{~m})$ & 3 \\
Data size (bytes) & 200 \\
$E_{\text {amp }}(\mathrm{J} / \mathrm{bit} / \mathrm{m})$ & $10^{-10}$ \\
$E_{\text {elec }}(\mathrm{n} J / \mathrm{bit})$ & 50 \\
\hline
\end{tabular}

following formula that takes into consideration the above parameters to proceed with paths classification:

$$
\begin{aligned}
& \alpha\left(\sum_{j=1}^{\operatorname{Size}\left(P_{i}\right)}(1-\beta)\left(\mathrm{Or}_{P_{j}}\left(n_{j}\right)\right)+\beta \sum_{k=1}^{r} \operatorname{Or}_{P_{k}}\left(n_{j}\right)\right) \\
& \quad+\gamma\left(\sum_{j=1}^{\operatorname{Size}\left(P_{i}\right)-1} d\left(s_{j}, s_{j+1}\right)\right) .
\end{aligned}
$$

The weighting factors $\alpha$ and $\gamma$ have been added to the equation to highlight the impact of each factor on the overall energy consumed, their corresponding values have been calculated based on the total energy consumed by a sensor node considering the parameters value presented in Table 2, and they are equal to

$$
\begin{aligned}
& \alpha=\frac{1}{10^{-10}\left(100+C_{r}^{2}\right)}, \\
& \gamma=\frac{C_{r}^{2}}{100+C_{r}^{2}},
\end{aligned}
$$

where

(i) $C_{r}$ is the maximum communication range;

(ii) $\beta$ is a variable indicating if the current node $n_{j}$ is an intersected node or not;

(iii) $\mathrm{Or}_{P_{k}}\left(n_{j}\right)$ indicates the order of $n_{j}$ in the path $P_{k}$;

(iv) $r$ is the number of paths that intersect at $n_{j}$ where $1 \leq$ $r \leq \mathrm{SN}$

(v) SN corresponds to the number of source nodes.

\section{Estimation of the Energy Consumption and the Network Lifetime}

In order to give an estimation of the energy consumed during a time interval $T$ (in $\mathrm{ms}$ ), we represent the number of events that occurred in a fixed interval $T$ with the Poisson distribution with parameter $\lambda$ :

$$
P(k, t)=\frac{(\lambda \cdot t)^{k}}{k !} \cdot e^{-\lambda \cdot t}
$$


TABLE 3: Simulation parameters.

\begin{tabular}{|c|c|c|c|c|}
\hline Realistic model & & & & \\
\hline \multirow{2}{*}{ Telos CC2420 } & RX MODES & & $62 \mathrm{mw}$ & \\
\hline & TX LEVELS & & $57.42 \mathrm{mw}$ & \\
\hline \multirow[t]{3}{*}{ Initial energy } & $18720(\mathrm{~J} / \mathrm{S})$ & & & \\
\hline & & $\mathrm{RX}$ & $\mathrm{TX}$ & $\overline{\text { SLEEP }}$ \\
\hline & $\mathrm{RX}$ & - & 62 & 62 \\
\hline \multirow[t]{2}{*}{ Transition power } & TX & 62 & - & 62 \\
\hline & SLEEP & 1.4 & 1.4 & - \\
\hline \multicolumn{5}{|l|}{ Theoretical model } \\
\hline$E_{\text {elec }}$ & $50 \mathrm{~nJ} / \mathrm{bit}$ & & & \\
\hline$E_{\text {amp }}$ & $100 \mathrm{pJ} / \mathrm{bit} / \mathrm{m}$ & & & \\
\hline$D$ & 200 bytes & & & \\
\hline$d$ & $3 \mathrm{~m}$ & & & \\
\hline
\end{tabular}

The estimated energy consumption for both realistic and theoretical model is given in

$$
\begin{aligned}
& E_{\text {node }_{i}}(\mathrm{RM})=\beta \cdot\left[\sum_{k=0}^{\alpha}\left(\frac{(\lambda \cdot t)^{k}}{k !} \cdot e^{-\lambda \cdot t}\right)\right. \\
& \left.\cdot\left((\alpha-k) \cdot E_{\mathrm{Rx}}+k \cdot E_{\mathrm{Tx}}+k\left(E_{\mathrm{RTT}}+E_{\mathrm{TTR}}\right)\right)\right]+\alpha \\
& \cdot E_{f} \\
& E_{\mathrm{node}_{i}}(\mathrm{TM})=\beta \cdot\left[( \sum _ { k = 0 } ^ { \alpha } \frac { ( \lambda \cdot t ) ^ { k } } { k ! } \cdot e ^ { - \lambda \cdot t } ) \cdot D \cdot \left(2 \cdot E_{\mathrm{elec}}\right.\right. \\
& \left.\left.+E_{\mathrm{amp}} \cdot d^{2}\right)\right]
\end{aligned}
$$

where

$$
\begin{aligned}
& \beta \in \mathbb{N}^{*}-\{1\}, \text { if } \text { node }_{i} \text { is an intersection point; } \\
& \beta=1 \text {, else } \\
& +E_{\mathrm{Tx}} \text { is energy of the transmission; } \\
& +E_{\mathrm{Rx}} \text { is energy of reception; } \\
& +E_{\mathrm{TTR}} \text { is energy of transition from TX to RX; } \\
& +E_{\mathrm{RTT}} \text { is energy of transition from RX to TX; } \\
& +E_{f} \text { is operating energy; } \\
& +k \text { is number of events occurrences, } k=0,1,2, \ldots ; \\
& +\alpha=\operatorname{Sup}\left(T / T_{u}\right) .
\end{aligned}
$$

When considering the realistic energy consumption model, the energy consumed when no event occurs during the time interval $T$ (which is represented in terms of time interval units $T_{u}$ ) depends mainly on the operating energy of the sensor node but also the energy of reception. When the transceiver transmit data for a long period of time, it consumes an important amount of energy required to transit from transmission to reception state and vice versa. For analysis purposes, we consider the simulations parameters depicted in Table 3. These parameters are defined in Castalia 2.3 , which is an open source simulator for WBSNs/WSNs [20-22]. The packet generation rate is constant and equals 5 pps (packets/sec). The initial energy level of each sensor node is assumed to be $18720 \mathrm{~J}$ and the data packet length is also constant and equals 200 bytes. The radio initial reception mode is set to high which stands for 1024 for data rate (kbps), DIFFQPSK as a modulation type, bandwidth $(\mathrm{MHz})$, sensitivity $(\mathrm{dBm})$, and power consumption (mW) equal, respectively, to $20,-87$, and 3.1. The main loop of Algorithm 1 is executed $\mathcal{O}(n)$ times. Choosing the set of paths to which the current path belongs requires time $\mathcal{O}(n)$. Algorithm 1 thus runs in time $\mathcal{O}\left(n^{2}\right)$.

6.1. Estimation of the Network Lifetime of a Nonintersecting Node in $T$ (ms). As can be seen in Figure 4, when the number of the occurred events increases, the corresponding network lifetime decreases, regardless of the average event occurrence value. These results were expected as the occurrence of an event puts the transceiver in transmission state after being transited from the reception state (in case of realistic modeling of the transceiver). Both radio transition and data transmission consume a considerable amount of energy as defined in the radio worksheet. Similarly to the theoretical model, more events occurrences lead to more bits transmitted in accordance with the distance. Therefore, an important amount of energy is consumed, which contributes to reducing the network lifetime. The network lifetime can be defined as the first sensor running out of energy or the $x \%$ sensor nodes dissipating their overall energy batteries. In our case of study, the dysfunction of the first sensor node defines the network lifetime in terms of intervals $T$ (100 s). Hence, in order to guarantee the best network performance, the routing functionality needs to include the fault tolerance option to avoid the frequent network topology reconfiguration and increase data packet delivery ratio, while minimizing the transmission delay.

6.2. Estimation of the Network Lifetime of an Intersecting Node in $T$ (ms). As depicted in Figure 5, there is a perfect correlation between the degree of the intersecting node and 


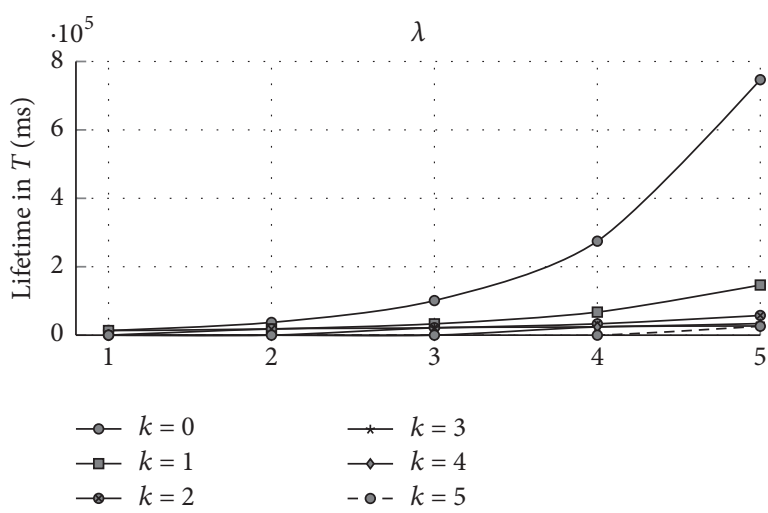

(a)

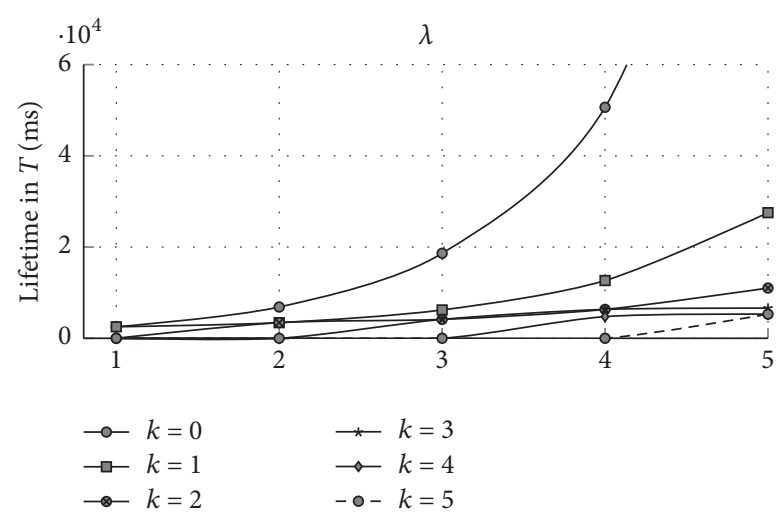

(b)

Figure 4: (a) Realistic model. (b) Theoretical model.

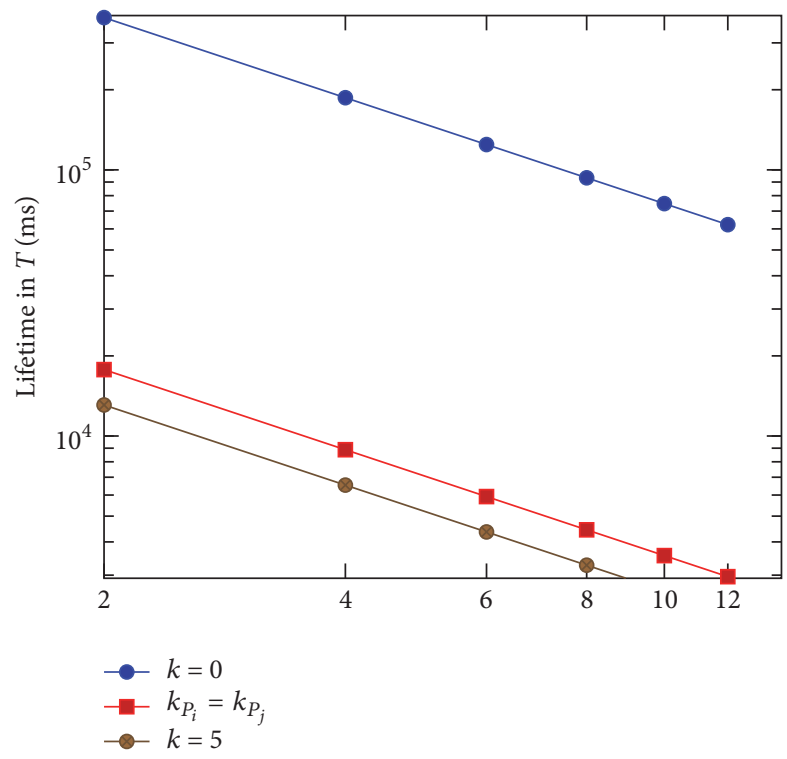

(a)

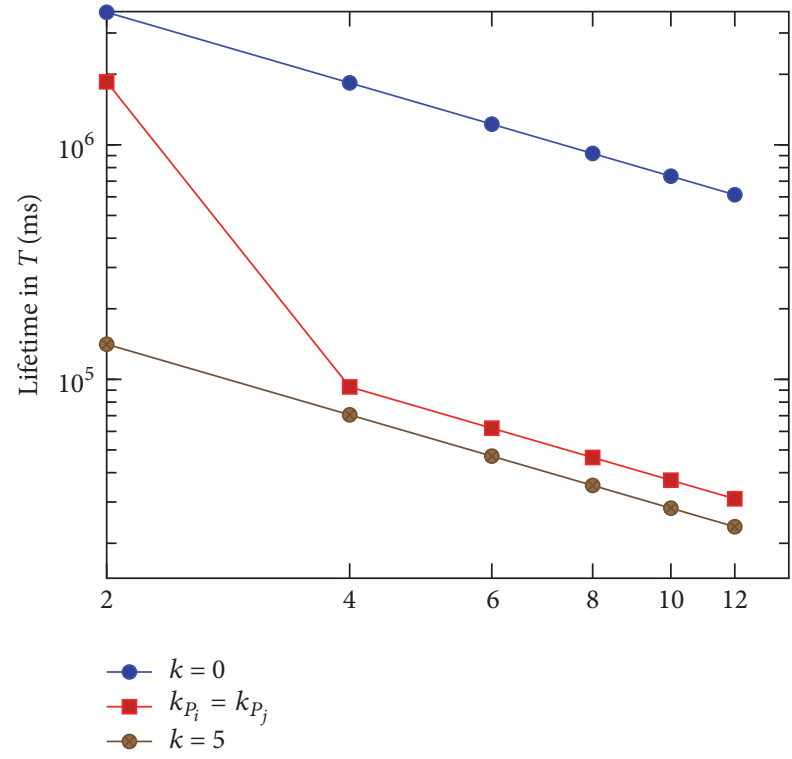

(b)

Figure 5: Degree of intersection node: (a) realistic model. (b) Theoretical model.

the network lifetime for both radio energy consumption models (realistic and theoretical). The bigger the degree of an intersecting point is, the less the network lifetime becomes. This tendency was expected as the intersecting point requires transmitting data packets which corresponds to zero (i.e., nonoccurrence of events) or many source nodes paths.

In our case of study, we did not consider a particular synchronization scenario where sensor nodes are active during a particular duty-cycle, after which the transceiver is turned off to save energy. In fact, the synchronization mechanism schema (when applied) makes a huge difference in energy consumption when both intersecting and nonintersecting nodes are considered. A nonintersecting node will spend more time in sleep mode, conserving though a great amount of energy, while an intersecting sensor node will have to stay on for most of the time as it delivers data to many source nodes, which leads to a rapid depletion of energy resource.
The number of event occurrence possibilities is equal to $\lambda^{\text {degree_point_inter }}$, where each path can deliver either $0,1, \ldots$ or $\lambda$ events.

\section{Multipaths or Optimal Path Routing?}

The proposed multipath approach equalized the energy consumed in the network. In Table 4, we compared our proposed routing approach with the optimal path routing in terms of network lifetime. To this end, we considered a grid network composed of 5 sensor nodes including two source nodes and the final destination. A random sensor deployment can also be utilized; however, it is important to employ additional number of relay sensors to insure the full network coverage. The results show that multipath routing outperforms the optimal path routing for both energy 
TABLE 4: Comparison of multipath and optimal path routing approaches.

\begin{tabular}{lcccc}
\hline & & Realistic model & \multicolumn{2}{c}{ Theoretical model } \\
& Multipath & Optimal path & Multipath & Optimal path \\
\hline Periodic & 1890 & 1804 & 415 & 320 \\
Random & 2712 & 2709 & 12572 & 9720 \\
\hline
\end{tabular}

consumption models and data collection type. However, the optimal path might show better results in case of random data collection, where any combination of source node paths can be taking place. This will increase the chance of utilizing a sensor node with a high intersection degree. Therefore, the sensor batteries will run out of energy in a very short amount of time, which leads to network dysfunction. It is very important to carry out a detailed performance analysis of both approaches before choosing the most suitable one for data routing. When the optimal path belongs to the $\mathrm{NIP}_{s}$ set, it is pointless to proceed with further analysis of lifetime estimation, as it is used by default in conjunction with other nonintersected paths. However, when it belongs to the $\mathrm{IP}_{s}$ set, the corresponding estimated lifetime will be compared with the multipath routing. If the latter presents the biggest value then the multipath approach is chosen, and the optimal path will be automatically used since it is considered as an intersected path. Else if the optimal path maximizes the network lifetime, then all the paths belonging to $\mathrm{IP}_{s}$, where the estimated network lifetime is inferior or equal to the optimal path one, will be used successively.

\section{Conclusion}

WBAN constitutes a subset of WSNs responsible for monitoring medical and nonmedical applications. WBAN consists in using biosensors to measure physiological parameters and forwarding them to the base station where further analyses are performed. This paper presents a new routing approach to increase the network lifetime by equalizing the energy consumption within sensor devices. The proposed approach takes into consideration the type of the radio model and the frequency of data collection. The statistical results prove the efficiency of our approach in minimizing the overall energy consumption. In fact, 89 time intervals were added to the network lifetime when a realistic model is applied, compared with 9815 in case of theoretical model. Hence, the frequent topology reconstruction is reduced, leading to a minimal network cost and transmission delay.

\section{Conflicts of Interest}

The authors declare that there are no conflicts of interest regarding the publication of this paper.

\section{References}

[1] Y. Sankarasubramaniam, I. F. Akyildiz, W. Su, and E. Cayirci, "Wireless sensor networks: a survey," Computer Networks, vol. 620, no. 1, pp. 393-422, 2002.
[2] P. P. Bhattacharya, J. Saraswat, and N. Rathi, "Techniques to enhance lifetime of wireless sensor networks: a survey," Global Journal of Computer Science and Technology (E), vol. 120, no. 1, 2012.

[3] J. Bergmann, K.-H. Wolf, O. J. Bott, M. Marschollek, U. Tegtbur, and R. Haux, "Sensor-enhanced health information system architectures for home and telecare: Concept and prototype," in Proceedings of the 2nd European Conference on eHealth, ECEH 2007, vol. 118, pp. 193-203, October 2007.

[4] N. Javaid, S. Faisal, Z. A. Khan, D. Nayab, and M. Zahid, "Measuring fatigue of soldiers in wireless body area sensor networks," in Proceedings of the 2013 IEEE 8th International Conference on Broadband, Wireless Computing, Communication and Applications, BWCCA 2013, pp. 227-231, Compiegne, France, October 2013.

[5] W. V. Shi and M. Zhou, "Body sensors applied in pacemakers: A survey," IEEE Sensors Journal, vol. 12, no. 6, pp. 1817-1827, 2012.

[6] P. A. Sandborn and P. Abshire, "2D motion sensor with programmable feature extraction," in Proceedings of the 2013 IEEE International Symposium on Circuits and Systems (ISCAS), pp. 2408-2411, Beijing, China, May 2013.

[7] B. D. Farnsworth, D. M. Talyor, R. J. Triolo, and D. J. Young, "Wireless in vivo EMG sensor for intelligent prosthetic control," in Proceedings of the TRANSDUCERS 2009 - 15th International Conference on Solid-State Sensors, Actuators and Microsystems, pp. 358-361, June 2009.

[8] M. P. urii, Z. Tafa, G. Dimi, and V. Milutinovi, A survey oF Military Applications of Wireless Sensor Networks, ISSN 2377$5475,2012$.

[9] S. K. Gupta, L. Schwiebert, Q. Tang, and N. Tummala, “TARA: Thermal-Aware Routing Algorithm for Implanted Sensor Networks," in Distributed Computing in Sensor Systems, vol. 3560 of Lecture Notes in Computer Science, pp. 206-217, Springer, Berlin, Germany, 2005.

[10] L. Rachid, N. El Moussaid, M. el Azhari, and A. Toumanari, "Relay based thermal aware and mobility support routing protocol for wireless body sensor networks," International Journal of Communication Networks and Information Security, vol. 80, no. 2, pp. 64-73, 2016.

[11] P. G. V. Naranjo, M. Shojafar, H. Mostafaei, Z. Pooranian, and E. Baccarelli, "P-sep: a prolong stable election routing algorithm for energy-limited heterogeneous fog-supported wireless sensor networks," The Journal of Supercomputing, vol. 730, no. 2, pp. 733-755, Feb 2017.

[12] C. Li, Z. Sun, H. Wang, and H. Song, "A novel energy-efficient $k$ Coverage algorithm based on probability driven mechanism of wireless sensor networks," International Journal of Distributed Sensor Networks, vol. 2016, Article ID 7474926, 9 pages, 2016.

[13] M. El azhari, A. Toumanari, and R. Latif, Performance Analysis of Multi-Hop and Cluster Based Routing Protocols for Mobile Heath Monitoring, 2014.

[14] Q. Sun, Y. Qiao, J. Wang, and S. Shen, "Node importance evaluation method in wireless sensor network based on energy 
field model," EURASIP Journal on Wireless Communications and Networking, vol. 2016, no. 1, 2016.

[15] S. Al-Janabi, I. Al-Shourbaji, M. Shojafar, and S. Shamshirband, "Survey of main challenges (security and privacy) in wireless body area networks for healthcare applications," Egyptian Informatics Journal, vol. 180, no. 2, pp. 113-122, 2016.

[16] S. Pirbhulal, H. Zhang, W. Wu, and Y. Zhang, "A Novel Biometric Algorithm to Body Sensor Networks," in Wearable Electronics Sensors, vol. 15 of Smart Sensors, Measurement and Instrumentation, pp. 57-79, Springer International Publishing, Cham, Germany, 2015.

[17] J. Chukwunonyerem, A. M. Aibinu, A. J. Onumanyi et al., "Development of key generation algorithm using ECG biometrics for node security in wireless body area sensor network," European Research in Telemedicine, vol. 5, no. 4, pp. 137-144, 2016.

[18] H. Balakrishnan, W. R. Heinzelman, and A. Chandrakasan, "Energy-efficient communication protocol for wireless microsensor networks," in Proceedings of the 33rd Annual Hawaii International Conference on System Siences (HICSS '00), vol. 2, IEEE, January 2000.

[19] Y. El Mourabit, A. Toumanari, M. El Azhari, and A. Bouirden, "Efficient energy mobile agent collector for intrusion detection system in wireless sensor network," Journal of Theoretical and Applied Information Technology, vol. 87, no. 3, pp. 520-526, 2016.

[20] D. Pediaditakis, Y. Tselishchev, and A. Boulis, "Performance and scalability evaluation of the Castalia wireless sensor network simulator," in Proceedings of the 3rd International ICST Conference on Simulation Tools and Techniques, SIMUTools 2010, pp. 53:1-53:6, March 2010.

[21] D. Benedetti, C. Petrioli, and D. Spenza, "GreenCastalia: an energy-harvesting-enabled framework for the castalia simulator," in Proceedings of the 1st ACM International Workshop on Energy Neutral Sensing Systems (ENSSys '13), pp. 7:1-7:6, ACM, Rome, Italy, November 2013.

[22] K. Lin, M. Chen, S. Zeadally, and J. J. P. C. Rodrigues, "Balancing energy consumption with mobile agents in wireless sensor networks," Future Generation Computer Systems, vol. 28, no. 2, pp. 446-456, 2012. 


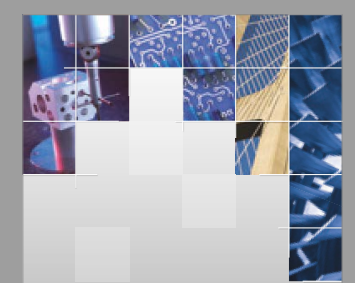

\section{Enfincering}
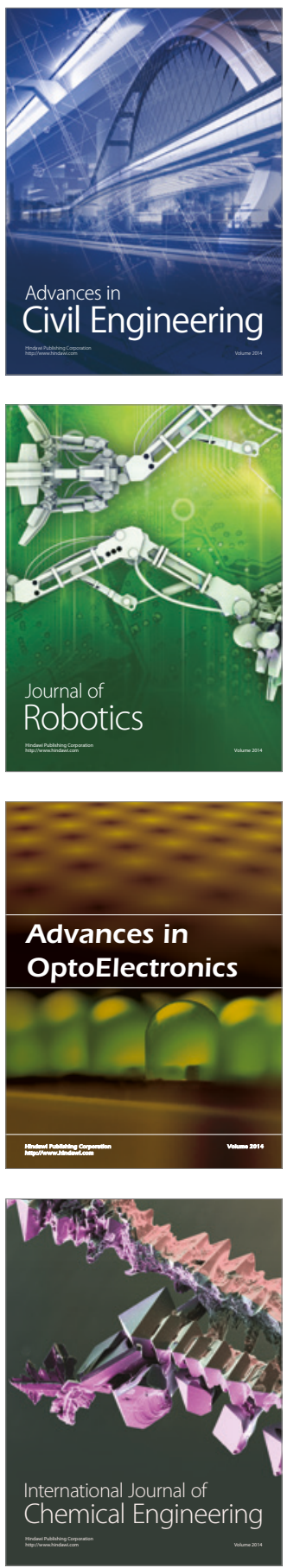

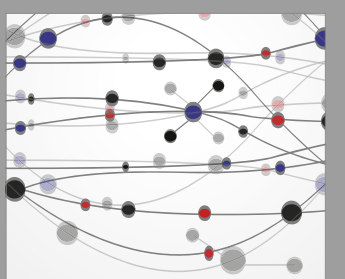

The Scientific World Journal

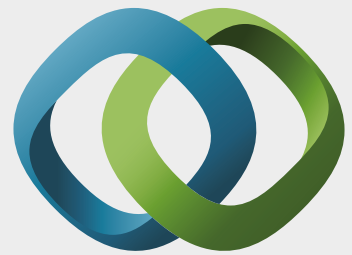

\section{Hindawi}

Submit your manuscripts at

https://www.hindawi.com
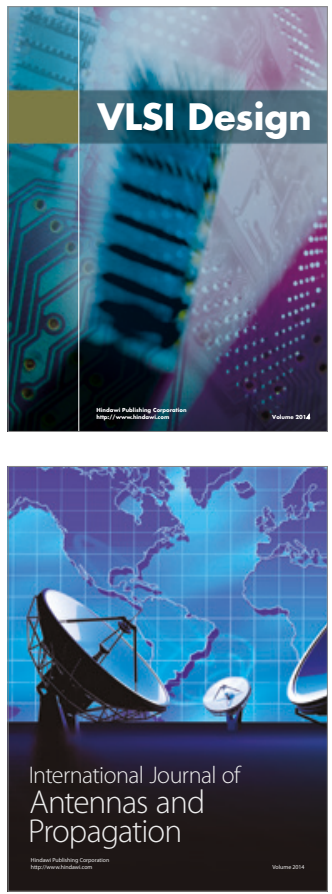

\section{Rotating}

Machinery
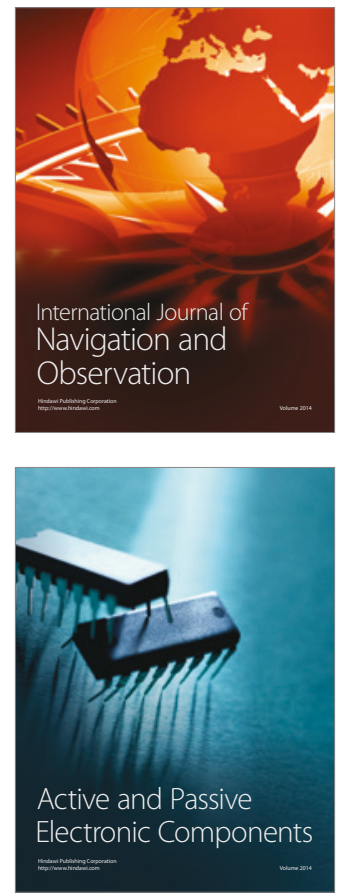
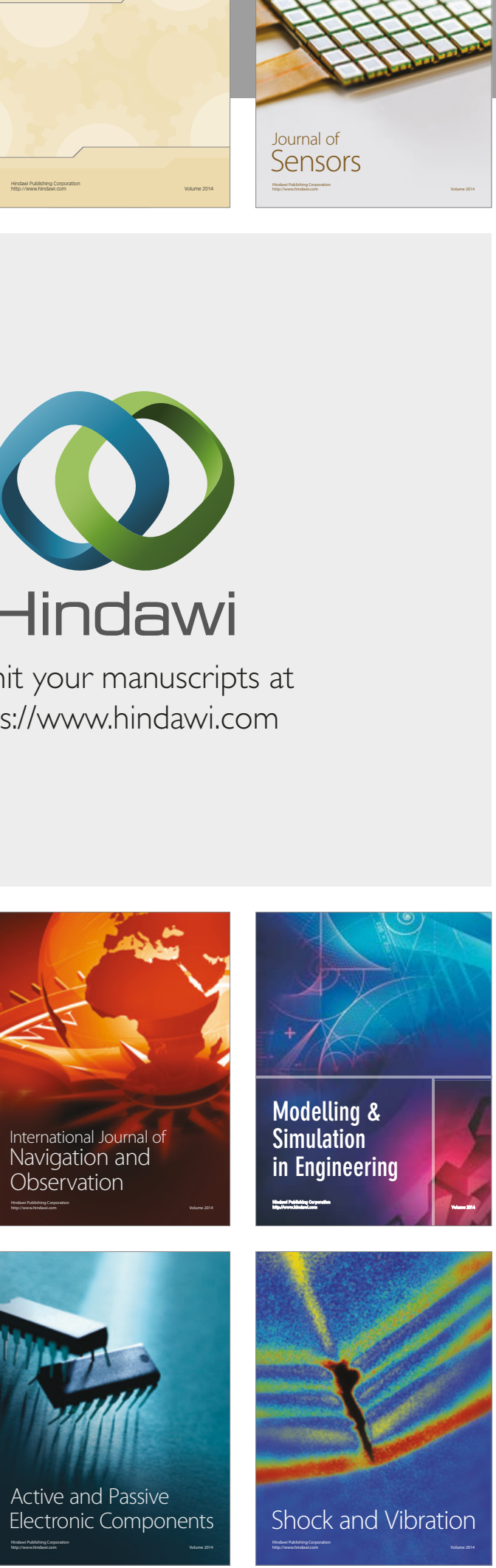
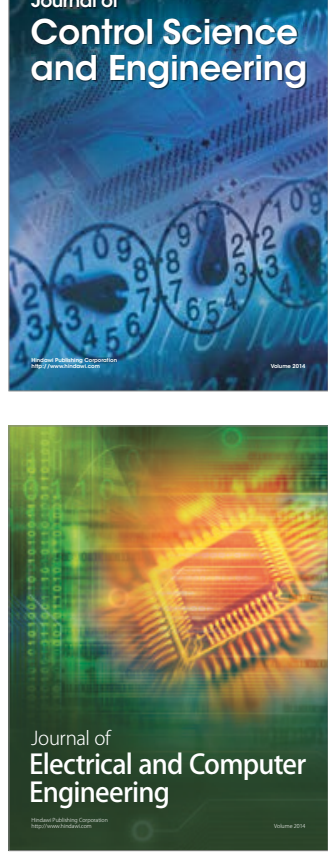

Distributed

Journal of

Control Science

and Engineering
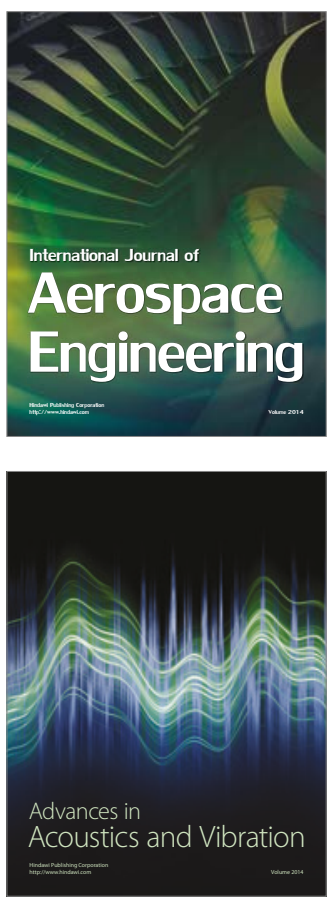

Sensor Networks 